\title{
Commentary Is CREB the Angry Bird that Releases Memory in Alzheimer's?
}

\author{
Allan Wang' and James A Bibb*,' \\ 'Departments of Psychiatry and Neurology and Neurotherapeutics, University of Texas Southwestern Medical Center, Dallas, TX, USA
}

Neuropsychopharmacology (201 I) 36, $2153-2154$; doi:10.1038/npp.201 I.126

In a now popular video game, pugnacious birds are catapulted at jumbled debris, with the goal of liberating their entrapped comrades. Analogously, potentiation of the cognition-mediating transcriptional factor, cyclic adenosine monophosphate (cAMP)-response element binding protein (CREB), or its downstream effectors, may release memory or cognitive function fettered by Alzheimer's disease pathology.

$\mathrm{AD}$ accounts for $50-60 \%$ of all cases of dementia, and currently affects 25 million people worldwide. The initial symptoms are cognitive deficits in several domains, including memory, language, and perceptual skills. Gross neuropathological changes such as the formation of extracellular amyloid plaques, composed of $\beta$-amyloid $(\mathrm{A} \beta)$ aggregates, and intracellular neurofibrillary tangles formed by hyperphosphorylated tau protein are the molecular hallmarks of $\mathrm{AD}$. Currently, there are no therapies to slow or otherwise halt the disruptions in neural communication that drive the progression of AD. Previous studies have linked $\mathrm{A} \beta$ to synaptic abnormalities and functional deficits associated with $\mathrm{AD}$ (Smith et al, 2009). Pathological aggregation of $\mathrm{A} \beta$ promotes synaptic dysfunction and disrupts activity-dependent gene transcription essential for learning and long-term memory formation. One such event in $\mathrm{AD}$ is the dysregulation of the transcription factor CREB. CREB signaling has a crucial role in orchestrating gene expression necessary for longterm synaptic plasticity and memory (Lopez de Armentia et al, 2007) and is highly conserved across species. Though the mechanisms underlying altered activity-dependent gene transcription in $\mathrm{AD}$ are largely unknown, selectively targeting $\mathrm{CREB}$ and its coactivators in $\mathrm{AD}$ patients presents a novel strategy for treating cognitive deficits. Inside this issue, Yiu et al, (2011) report the study of mice that express a human $\beta \mathrm{APP}_{695}$ transgene with familial $\mathrm{AD}$ mutations, resulting in $\mathrm{A} \beta$ pathology in the dorsal hippocampus characteristic of early-onset $\mathrm{AD}$. These mice exhibited pronounced spatial memory impairment.

*Correspondence: Dr JA Bibb, Department of Psychiatry and Neurology and Neurotherapeutics, University of Texas Southwestern Medical Center, 5323 Harry Hines Boulevard, NC 5.4I0, Dallas, TX, 75390, USA, Tel: +2 14648 4I68, Fax: +2 14648 1293, E-mail: james.bibb@utsouthwestern.edu

Received 06 June 201 I; accepted 9 June 201 I
The CA1 region of the dorsal hippocampus is among the first to exhibit $\mathrm{AD}$ pathology in humans. In particular, the CA1 region, in both human patients and mouse $\mathrm{AD}$ models, exhibits deficits in CREB activation, neuronal complexity, and neuronal network activity. The authors postulated that locally and acutely increasing CREB levels in the CA1 region through CREB vector microinjection should restore function in all three domains. Indeed, the rescue was successful, both in terms of facilitating long-term allocentric spatial memory, and reversing the deficits in dendritic arborization and neuronal network activity. This was presumably accomplished by restoring phosphorylated CREB to a level that allowed re-initiation of activity-dependent gene transcription. The results of this rescue closely mirror those of previous studies in which synaptic pathology was reversible by restoring CREB function, through agents such as rolipram (Smith et al, 2009). The findings are further validated by the observation that the rescue of spatial memory was anatomically specific to the CA1. The authors highlight the fact that improvements to spatial memory deficits occurred independent of $\mathrm{A} \beta$ plaque load. Thus, increasing CREB in the CA1 region does not improve spatial memory through a mechanism of $\mathrm{A} \beta$ clearance. This coincides with other studies that suggest the extent of synapse loss is a more robust correlate of cognitive impairment in $\mathrm{AD}$ patients than that of either $\beta$-amyloid or neurofibrillary tangle pathology (Coleman and Yao, 2003). Perhaps strategies that enhance the CREB signaling cascades can restore learning and memory through compensatory mechanisms that circumvent $\beta$-amyloid pathology (Figure 1).

$\mathrm{A} \beta$ remains a subject of intense research not only as a potential marker in early diagnosis, but also for its role in dysregulating excitatory activity-dependent receptors and upstream activators of CREB. Amyloid deposition also attenuates CREB activity through its coactivators, CREBbinding protein (Francis et al, 2006), and CRTC1 (España et al, 2010). Notably, the downregulation of CRTC1mediated CREB transcription by $\mathrm{A} \beta$ disrupts neuronal expression of genes related to synaptic plasticity such as c-fos, Bdnf IV, and Nr4a2 (España et al, 2010). Although the selective targeting of CREB may transiently enhance allocentric spatial memory by increasing synaptic plasticity, it remains to be seen whether chronic CREB therapy in later 


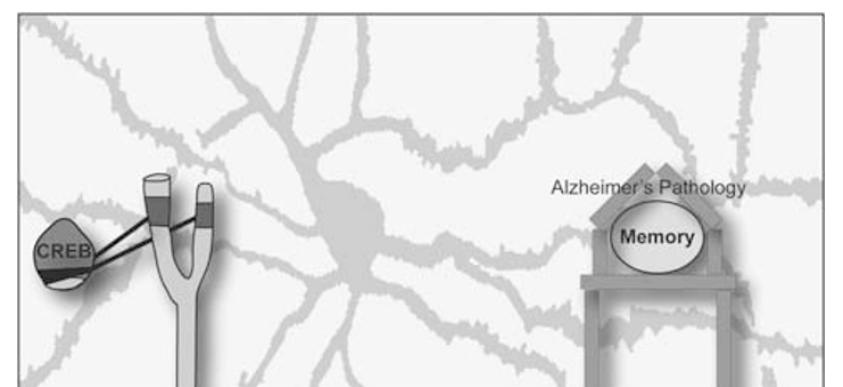

Figure I Cyclic adenosine monophosphate (CAMP)-response element binding protein (CREB) restores cognition in Alzheimer's disease (AD) model mice.

stages will be beneficial if $\mathrm{A} \beta$ deposition remains unchecked. The potential of CREB-mediated pathways, known and unknown, to serve as novel pharmaceutical targets for $\mathrm{AD}$ is compelling. However, attaining the same degree of targeting specificity in $\mathrm{AD}$ patients through intrahippocampal injections may prove impractical as a long-term and widespread treatment. The authors note that translating these findings will require a greater understanding of CREBmediated changes in neuronal morphology, as well as any deleterious effects from chronic upregulation of CREB expression. In fact, previous studies have shown that sustained overexpression of CREB in the CA1 region in mice leads to a significant loss of hippocampal neurons and sporadic epileptic seizures (Lopez de Armentia et al, 2007).

Throughout the exploration of novel therapeutic strategies, it is important to acknowledge the limitations of familial, early-onset mouse models of AD. Sporadic AD represents the vast majority (90-95\%) of cases. Although current $\mathrm{AD}$ mouse models approximate the amalgamation of neuropathological insults with great fidelity, perhaps this approach does not encompass the entire scope of agerelated cognitive decline. For example, a recent study demonstrated that histone acetylation in adult mice relative to their juvenile counterparts is reduced, thus resulting in deficient learning-induced gene expression (Peleg et al, 2010). Furthermore, targeting epigenetic mechanisms may allow recovery of lost long-term memories via synaptogenesis and neural network rewiring (Fischer et al, 2007). Further measures linking AD mouse models to human cohorts are necessary to provide mechanistic connections between aging and neurodegenerative disorders. Ultimately, a deeper knowledge of the role of CREB, and how its downstream targets mediate synaptic remodeling, will spur the development of novel therapeutic approaches in the prevention and amelioration of cognitive decline in Alzheimer's patients.

\section{ACKNOWLEDGEMENTS}

We thank Florian Plattner for helpful suggestions in preparing this commentary. This work was supported by NIH support from the National Institute of Mental Health, R01MH083711.

\section{DISCLOSURE}

The authors declare no conflict of interest.

\section{REFERENCES}

Coleman PD, Yao PJ (2003). Synaptic slaughter in Alzheimer's disease. Neurobiol Aging 24: 1023-1027.

España J, Valero J, Miñano-Molina AJ, Masgrau R, Martin E, Guardia-Laguarta C et al. (2010). $\beta$-Amyloid disrupts activitydependent gene transcription required for memory through the CREB coactivator CRTC1. J Neurosci 30: 9402-9410.

Fischer A, Sananbenesi F, Wang X, Dobbin M, Tsai LH (2007). Recovery of learning and memory is associated with chromatin remodeling. Nature 447: 178-182.

Francis YI, Stephanou A, Latchman DS (2006). CREB binding protein activation by presenilin 1 but not by its M146L mutant. Neuroreport 17: 917-921.

Lopez de Armentia M, Jancic D, Olivares R, Alarcon JM, Kandel ER, Barco A (2007). cAMP response element-binding protein-mediated gene expression increases the intrinsic excitability of CA1 pyramidal neurons. J Neurosci 27: 13909-13918.

Peleg S, Sananbenesi F, Zovoilis A, Burkhardt S, Bahari-Javan S, Agis-Balboa RC et al. (2010). Altered histone acetylation is associated with age-dependent memory impairment in mice. Science 328: 753-756.

Smith DL, Pozueta J, Gong B, Arancio O, Shelanski M (2009). Reversal of long-term dendritic spine alterations in Alzheimer disease models. Proc Natl Acad Sci USA 106: 16877-16882.

Yiu AP, Rashid AJ, Josselyn SA (2011). Increasing CREB function in the CA1 region of dorsal hippocampus rescues the spatial memory deficits in a mouse model of Alzheimer's disease. Neuropsychopharmacology 36: 2169-2186. 